Sergio Conti · Georg Dolzmann · Bernd Kirchheim · Stefan Müller

\title{
Sufficient conditions for the validity of the Cauchy-Born rule close to $\mathrm{SO}(n)$
}

Received September 25, 2005

\begin{abstract}
The Cauchy-Born rule provides a crucial link between continuum theories of elasticity and the atomistic nature of matter. In its strongest form it says that application of affine displacement boundary conditions to a monatomic crystal will lead to an affine deformation of the whole crystal lattice. We give a general condition in arbitrary dimensions which ensures the validity of the Cauchy-Born rule for boundary deformations which are close to rigid motions.

This generalizes results of Friesecke and Theil [J. Nonlin. Sci. 12 (2002), 445-478] for a twodimensional model. As in their work, the key idea is to use a discrete version of polyconvexity (ordinary convexity of the elastic energy as a function of the atomic positions is ruled out by frame indifference). The main point is the construction of a suitable discrete null Lagrangian which allows one to separate rigid motions. To do so we observe a simple identity for the determinant function on $\mathrm{SO}(n)$ and use interpolation to convert ordinary null Lagrangians into discrete ones.
\end{abstract}

Keywords. Cauchy-Born rule, atomistic models, null Lagrangian

\section{Introduction}

The continuum theory of elasticity has been very successful in the study of crystalline microstructure [BJ87, CK88, Mü99, JH00, Bh03, Do03]. The link between the continuum theory and the atomistic nature of matter is usually made by an appeal to the CauchyBorn rule (also called Cauchy-Born hypothesis or Born rule; see, e.g., the discussion in [Er82, PZ03]). It states, loosely speaking, that a macroscopic affine deformation $x \mapsto F x$ corresponds to an affine deformation of the individual atomic positions. In the context of solid-solid phase transformations there is an extensive literature on the validity and relevance of the Cauchy-Born rule (see [Pa80, Er82, Za92, Er97, FT02, PZ03, CZ04, BCZZ04] and the references therein), mostly based on symmetry considerations. Until

S. Conti: Fachbereich Mathematik, Universität Duisburg-Essen, Lotharstr. 65, 47057 Duisburg, Germany; e-mail: conti@math.uni-duisburg.de

G. Dolzmann: Mathematics Department, University of Maryland, College Park, MD 20742-4015, USA; e-mail: dolzmann@math.umd.edu

B. Kirchheim: University of Oxford, Mathematical Institute, 24-29 St Giles', Oxford, OX1 3LB, UK; e-mail: kirchhei@maths.ox.ac.uk

S. Müller: Max Planck Institute for Mathematics in the Sciences, Inselstr. 22-26, 04103 Leipzig, Germany; e-mail: sm@mis.mpg.de

Mathematics Subject Classification (2000): 74B20, 74N05 
very recently, however, there has been no analysis of the validity of the Cauchy-Born rule starting from a suitable atomistic theory. In this context we interpret the CauchyBorn rule in the spirit of [FT02] in the following way. Consider a Bravais lattice $\mathcal{L} \subset \mathbb{R}^{n}$. To a subset $\Lambda \subset \mathcal{L}$ and a deformation $y: \Lambda \rightarrow \mathbb{R}^{n}$ we associate an elastic energy $E_{\Lambda}(y)$. We say that the Cauchy-Born rule holds for a matrix $F$ if for all bounded $\Lambda$ the minimizer of $E_{\Lambda}(y)$ subject to the affine boundary condition

$$
y(x)=F x \quad \text { for } x \in \partial \Lambda
$$

is given by the affine deformation $y(x)=F x$, for all $x \in \Lambda$ (see (2.1) and 2.2) below for the precise definition of the boundary $\partial \Lambda$ ). In the case of multilattices, this corresponds to focussing on the skeletal lattice, with an energy $E_{\Lambda}$ which has already been minimized over the motif (i.e., the displacements of the atoms inside the unit cell).

Friesecke and Theil [FT02] studied a two-dimensional mass-spring model with nearest and next nearest neighbour interactions and showed that the Cauchy-Born rule holds (in the above sense) for a suitable range of parameters and for $F$ which are close to a rotation $R \in \mathrm{SO}(2)$. Such a stability result is remarkable since a realistic atomistic energy cannot be a convex function of $y$, in view of frame indifference (i.e., invariance under rotations). The main point is to show that if the energy of a single cell is minimized at the identity and is positive definite transversal to rigid motions then the Cauchy-Born rule holds for all $F$ near the identity.

Here we extend these results to general dimensions and to general finite range massspring models (see Theorems 4.2 and 5.1 below). As in [FT02] the main point is, roughly speaking, to construct a suitable discrete null Lagrangian, i.e., a discrete energy $N(y)$ which depends only on the boundary values, such that $E+N$ always lies above a convex function $H$ and equals $H$ if $y(x)=F x$ (by discrete energy we mean an energy defined on the discrete space, in contrast to the continuum energy which is defined on vector fields). Our construction relies on two ingredients: first, a systematic way to obtain discrete null Lagrangians from ordinary ones by interpolation (see Theorem 3.2 below), and secondly, a simple, yet powerful identity to separate points on $\operatorname{SO}(n)$ by a continuous null Lagrangian (see (H5') in Section 4 below). Then one can easily conclude by a perturbation and compactness argument. In this way one also obtains a slightly streamlined version of the original proof in [FT02].

Finally, we briefly comment on the interpretation of the Cauchy-Born rule discussed above and the relevance of mass-spring models. The above form of the Cauchy-Born rule is rather stringent. Since we require the affine map to be the unique minimizer for affine boundary conditions for arbitrarily large subsets $\Lambda$ we effectively exclude both atomistic and mesoscopic oscillations (e.g., twinning). A weaker requirement would be obtained by restricting the condition to sets $\Lambda$ which are small multiples of the lattice unit cell. However, there does not seem to be a natural choice of the cut-off length. This is related to the question of whether a certain nonaffine minimizing arrangement should be considered a phase (i.e., an atomistic pattern with a possibly large unit cell) or whether it should be considered a microstructure (i.e., a mesoscopic mixture of different phases).

In the mass-spring models the type of interaction between different atoms (e.g., nearest neighbour, next-nearest neighbour, ...) is determined by the position of the atoms in the 
reference lattice $\mathcal{L}$. Thus the energy is not invariant under the noncompact group $\operatorname{GL}(\mathbb{Z}, 3)$ of lattice-preserving linear transformations. If one includes invariance under all elements in $\operatorname{GL}(\mathbb{Z}, 3)$, then a crystal has no resistance to macroscopic shears, at least in a purely static variational approach as considered in this paper (for a sharp result in the continuum setting see [Fo87]). Sheared states do not correspond to energy minimizers but rather to metastable states. A full analysis of their behaviour (which would have to include a detailed discussion of the nucleation and motion of dislocations and other lattice defects) seems currently to be out of reach. The mass-spring models stabilize the metastable state by fixing the neighbourhood relations and imposing suitable growth conditions on the potential (see, e.g., hypothesis (H6) in Section 4 below) which enforce that, given suitable energy bounds, the local structure of the reference lattice is preserved, at least near most points. For uniformly small deformation gradients, E and Ming [EM05] have studied the relation between the time-dependent discrete and continuous equations under suitable ellipticity assumptions on the continuous energy density and assumptions on the phonon spectrum of the discrete model.

\section{Notation and assumptions on the cell energy}

Throughout this paper we assume that the underlying lattice is given by $\mathcal{L}=\mathbb{Z}^{n}$, i.e., the lattice is cubic with lattice parameter equal to one (we could equally well consider a general Bravais lattice, but we prefer the cubic lattice for notational convenience). Let $x_{1}, \ldots, x_{2^{n}}$ be the enumeration of the vertices of the unit cube $[0,1]^{n}$ such that the coordinates of $x_{i}$ in the standard basis of $\mathbb{R}^{n}$ correspond to the digits in the binary representation of $i-1$, i.e., $i-1=\sum_{j=1}^{n}\left(x_{i}\right)_{j} 2^{j-1}$. In particular, $x_{1}$ corresponds to the origin. For simplicity we write $\mathbf{x}=\left(x_{1}, \ldots, x_{2^{n}}\right) \in\left(\mathbb{R}^{n}\right)^{2^{n}} \sim \mathbb{R}^{n \times 2^{n}}$. Finally, it is convenient to introduce the mapping $\pi: \mathbb{R}^{n} \rightarrow \mathbb{R}^{n \times 2^{n}}$ by $\boldsymbol{\pi}(c)=(c, \ldots, c)$. Thus $\mathrm{x}+\boldsymbol{\pi}(c)=\left(x_{1}+c, \ldots, x_{2^{n}}+c\right)$ describes a translation of the unit cell by the vector $c$. In our analytical calculations we are basically interested in the orthogonal complement of the space $V_{0}:=\pi\left(\mathbb{R}^{n}\right)$ of all such shift directions.

If $A \in \mathbb{M}^{n \times n}$ represents a linear mapping from $\mathbb{R}^{n}$ into $\mathbb{R}^{n}$, then we set $A \mathbf{x}=$ $\left(A x_{1}, \ldots, A x_{n}\right)$.

For a subset $\Lambda$ of points in $\mathcal{L}$ we define the set of interior points

$$
\Lambda^{\circ}=\left\{x \in \Lambda: x-x_{i} \in \Lambda \text { for } i=1, \ldots, 2^{n}\right\} .
$$

The reason for this particular choice will become clear in the proof of Theorem 3.2. It is also convenient to consider Dirichlet boundary conditions to be given on

$$
\partial \Lambda=\mathcal{L} \backslash \Lambda^{\circ},
$$

i.e., we consider deformations that are local perturbations of a given deformation of the lattice. Here an elastic deformation of the lattice is a map $y: \mathcal{L} \rightarrow \mathbb{R}^{n}$ that assigns to each point $x \in \mathcal{L}$ a new position $y(x)$ in the deformed lattice. We call the vector $D^{\prime} y(x)$ defined by

$$
D^{\prime} y(x)=\left(y\left(x+x_{1}\right)-\bar{y}(x), \ldots, y\left(x+x_{2^{n}}\right)-\bar{y}(x)\right)=\mathbf{y}-\overline{\mathbf{y}}
$$


the discrete gradient of $y$. Here

$$
\bar{y}(x)=\frac{1}{2^{n}} \sum_{i=1}^{2^{n}} y\left(x+x_{i}\right) \quad \text { and } \quad \overline{\mathbf{y}}=\boldsymbol{\pi}(\bar{y}(x)) .
$$

For affine deformations $y(x)=F x$ of the lattice we define

$$
F^{\prime}=D^{\prime} y=F(\mathbf{x}-\overline{\mathbf{x}}) .
$$

Notice that the subspace of discrete gradients coincides with the orthogonal complement of the shift directions in $\mathbb{R}^{n \times 2^{n}}$.

The energy that is required to deform a unit cell in the lattice by mapping the corners $x_{i}$ to the new positions $y_{i}$ is given by $E_{\text {cell }}(\mathbf{y})$, where $E_{\text {cell }}$ is a mapping from $\mathbb{R}^{n \times 2^{n}}$ into $\mathbb{R}$. Lattice deformations that correspond to rigid rotations will be important in the proof of the validity of the Cauchy-Born rule, and we define therefore

$$
\mathrm{SO}(n)^{\prime}=\left\{R^{\prime} \in \mathbb{R}^{n \times 2^{n}}: \exists R \in \mathrm{SO}(n) \text { such that } R^{\prime}=R(\mathbf{x}-\overline{\mathbf{x}})\right\} .
$$

We assume that $E_{\text {cell }}$ has the following properties:

(H1) Invariance under rotations and translations: We have

$$
E_{\text {cell }}(R \mathbf{y})=E_{\text {cell }}(\mathbf{y}) \quad \text { for all } R \in \mathrm{SO}(n)
$$

and

$$
E_{\text {cell }}(\mathbf{y}-\boldsymbol{\pi}(c))=E_{\text {cell }}(\mathbf{y}) \quad \text { for all } c \in \mathbb{R}^{n} .
$$

(H2) Characterization of the ground state: We have $E_{\text {cell }} \geq 0$ and $E_{\text {cell }}(\mathbf{y})=0$ if and only if $\mathbf{y}$ corresponds to a rigid body rotation, i.e., there exist $R \in \mathrm{SO}(n)$ and $c \in \mathbb{R}^{n}$ such that $\mathbf{y}=R \mathbf{x}+\boldsymbol{\pi}(c)$.

(H3) Smoothness and convexity: $E_{\text {cell }}$ is smooth in a neighbourhood of $\mathrm{SO}(n)^{\prime}$ and the Hessian $D^{2} E_{\text {cell }}$ at the identity $I^{\prime}$ is positive definite on the orthogonal complement of the subspace spanned by all shift directions $\pi(c)$ and infinitesimal rotations $W^{\prime}$ (which correspond to skew-symmetric affine deformations, $W^{T}=-W$ ).

(H4) Growth condition at infinity: We assume that

$$
\liminf _{G^{\prime} \in V_{0}^{\perp}, G^{\prime} \rightarrow \infty} \frac{E_{\text {cell }}\left(G^{\prime}\right)}{\left|G^{\prime}\right|^{n}}>0,
$$

where $V_{0}$ is the space $\boldsymbol{\pi}\left(\mathbb{R}^{n}\right)$ of shift directions.

Note that in view of $(\mathrm{H} 1)$ we can define $E_{\text {cell }}$ as a function of the discrete gradient, $E_{\text {cell }}(\mathbf{y})=E_{\text {cell }}(\mathbf{y}-\overline{\mathbf{y}})=E_{\text {cell }}\left(D^{\prime} y\right)$. 


\section{Discrete null Lagrangians}

Null Lagrangians play an important role in the proof of uniqueness of minimizers subject to affine boundary conditions, and it is not surprising that an analogue of this definition for lattice functions will prove pivotal in the verification of the Cauchy-Born rule.

Recall that a function $N: \mathbb{M}^{m \times n} \rightarrow \mathbb{R}$ is called a null Lagrangian if for all $F \in$ $\mathbb{M}^{m \times n}$ and for all $\phi \in W_{0}^{1, \infty}\left(\Omega ; \mathbb{R}^{m}\right)$,

$$
\int_{\Omega} N(F) d x=\int_{\Omega} N(F+D \phi) d x,
$$

where $\Omega$ is an open and bounded domain. Equivalently, null Lagrangians can be defined as those functions $N$ for which both $N$ and $-N$ are quasiconvex in the sense of Morrey [Mo52]. A classical result states that $N$ is a null Lagrangian if and only if it is an affine combination of minors (subdeterminants; see the appendix for further details). The same characterization holds for null Lagrangians involving higher derivatives but the argument is much more subtle [BCO81].

We now define the analogue of null Lagrangians for lattice functions.

Definition 3.1. A mapping $N^{\prime}: \mathbb{R}^{n \times 2^{n}} \rightarrow \mathbb{R}$ is called a discrete null Lagrangian if for every finite subset $\Lambda \subset \mathcal{L}$ and every $F \in \mathbb{M}^{n \times n}$,

$$
\sum_{x \in \Lambda} N^{\prime}\left(D^{\prime} y(x)\right)=\sum_{x \in \Lambda} N^{\prime}\left(F^{\prime}\right)
$$

whenever $y(x)=F x$ for all $x \in \mathcal{L} \backslash \Lambda^{\circ}$.

The crucial observation explained below is that any null Lagrangian in the continuous setting induces in a natural way a discrete null Lagrangian. This will give us sufficiently many null Lagrangians for our uniqueness result to follow.

It should, however, also be mentioned that not all discrete null Lagrangians can be found this way. Indeed, for $n=2$ the discrete null Lagrangian $N^{\prime}\left(x_{1}, x_{2}, x_{3}, x_{4}\right)=$ $\left(x_{2}-x_{1}\right)_{1}$ cannot be obtained by linear interpolation from a null Lagrangian since for cell deformations with $x_{1}-x_{2} \neq x_{3}-x_{4}$ incompatibilities do occur.

Theorem 3.2. Let $N: \mathbb{M}^{n \times n} \rightarrow \mathbb{R}$ be a null Lagrangian. Then there exists a discrete null Lagrangian $N^{\prime}: \mathbb{R}^{n \times 2^{n}} \rightarrow \mathbb{R}$ which is invariant under translation and agrees with $N$ on all Cauchy-Born deformations, i.e.,

$$
N^{\prime}\left(F^{\prime}\right)=N(F)
$$

where $F \in \mathbb{M}^{n \times n}$ and $F^{\prime}=F(\mathbf{x}-\overline{\mathbf{x}})$ is the discrete gradient. Moreover, $N^{\prime}$ is a polynomial of the same degree as $N$. 
Proof. The discrete null Lagrangian can be constructed from the given null Lagrangian $N$ by extending a lattice deformation on $\mathcal{L}$ to a deformation defined on $\mathbb{R}^{n}$. A convenient way to do this is by multilinear interpolation. We describe the construction for the unit cell of the lattice; the construction on any other cell in the lattice is analogous.

Suppose that $y_{i}=y\left(x_{i}\right)$ are the values of the deformation at the corners of the unit cell in the lattice. Then we define the extension $\tilde{y}$ as the unique function from the unit cube $[0,1]^{n}$ into $\mathbb{R}^{n}$ that is affine in each coordinate direction and satisfies the interpolation conditions $\tilde{y}\left(x_{i}\right)=y_{i}$. The map $\mathbf{y} \mapsto \tilde{y}$ is linear and one-to-one. Moreover, if the lattice deformation is compatible with an affine deformation, i.e., if there exist $F \in \mathbb{M}^{n \times n}$ and $c \in \mathbb{R}^{n}$ such that $\mathbf{y}=F \mathbf{x}+\boldsymbol{\pi}(c)$, then $\tilde{y}(x)=F x+c$ for all $x \in \bar{Q}$ were $Q=(0,1)^{n}$. Finally, note that the function $\tilde{y}: \mathbb{R}^{n} \rightarrow \mathbb{R}^{n}$ that is obtained by constructing the extension locally in each cell of the lattice, is globally continuous and locally Lipschitz continuous. This follows from the fact that the values of the interpolation $\tilde{y}$ restricted to one face of the cube depend only on the values $y_{i}=y\left(x_{i}\right)$ at the vertices $x_{i}$ contained in this face.

We now define

$$
N^{\prime}(\mathbf{y})=\int_{Q} N(\nabla \tilde{y}(x)) d x .
$$

With this definition, (3.1) follows immediately from the compatibility of the extension with affine functions. Since $N$ is a polynomial and the map $\mathbf{y} \mapsto \nabla \widetilde{y}$ is linear, $N^{\prime}$ is a polynomial with $\operatorname{deg} N^{\prime} \leq \operatorname{deg} N$ and in view of (3.1) the degrees must be equal.

To see that $N^{\prime}$ is a discrete null Lagrangian, consider a lattice function $y: \mathcal{L} \rightarrow \mathbb{R}^{n}$ and the associated multilinear extension $\tilde{y}: \mathbb{R}^{n} \rightarrow \mathbb{R}^{n}$. Let $\Lambda \subset \mathcal{L}$ and suppose that $y(x)=F x$ for $x \in \mathcal{L} \backslash \Lambda^{\circ}$. Then

$$
\sum_{x \in \Lambda} N^{\prime}\left(D^{\prime} y\right)=\sum_{x \in \Lambda} \int_{x+Q} N(\nabla \tilde{y}) d x=\int_{U} N(\nabla \tilde{y}) d x
$$

where $U=\bigcup_{x \in \Lambda}(x+\bar{Q})$. The boundary of $U$ consists therefore of faces $F_{j}$ of unit cubes contained in the lattice, and by definition no vertex in $\Lambda^{\circ}$ can be contained in $\partial U$. Therefore $\tilde{y}(x)=F x$ on $\partial U$, and since $N$ is a null Lagrangian,

$$
\int_{U} N(\nabla \widetilde{y}) d x=\int_{U} N(F) d x=\sum_{x \in \Lambda} N(F)=\sum_{x \in \Lambda} N^{\prime}\left(F^{\prime}\right) .
$$

The foregoing two identities imply the assertion of the theorem.

\section{Validity of the Cauchy-Born rule}

In this section we establish the validity of the Cauchy-Born rule for lattice deformations that are close to deformations that correspond to rigid rotations. The key ingredient is the existence of a discrete null Lagrangian $N^{\prime}$ with the following two properties that will be important in the proof: 
(H5) There exists a constant $c>0$ such that

$$
N^{\prime}\left(H^{\prime}\right)-N^{\prime}\left(G^{\prime}\right)-\nabla N^{\prime}\left(G^{\prime}\right):\left(H^{\prime}-G^{\prime}\right) \geq c\left|H^{\prime}-G^{\prime}\right|^{2}
$$

for all $G^{\prime}, H^{\prime} \in \mathrm{SO}(n)^{\prime}$.

(H6) We have

$$
\limsup _{H^{\prime} \in V_{0}^{\perp}, H^{\prime} \rightarrow \infty} \frac{\left|N^{\prime}\left(H^{\prime}\right)\right|+\left|D N^{\prime}\left(H^{\prime}\right) H^{\prime}\right|}{\left|H^{\prime}\right|^{n}}<\infty .
$$

It turns out that in fact the determinant of an $n \times n$ matrix is sufficient to construct a discrete null Lagrangian with the foregoing properties.

Proposition 4.1. Let $N^{\prime}$ be the discrete null Lagrangian constructed from $N(F)=\operatorname{det} F$ for $F \in \mathbb{M}^{n \times n}$ using Theorem 3.2. Then $N^{\prime}$ satisfies (H5) and (H6).

Proof. First we observe that $N$ itself satisfies the corresponding identity

$\left(\mathrm{H}^{\prime}\right) N(H)-N(G)-\nabla N(G):(H-G)=\frac{1}{2}|H-G|^{2}$ if $G, H \in \mathrm{SO}(n)$.

Indeed, since $\nabla N(G)=\operatorname{cof} G$ for all $G \in \mathbb{M}^{n \times n}$ and $\operatorname{cof} G=G$ on $\operatorname{SO}(n)$ the left hand side of $\left(\mathrm{H}^{\prime}\right)$ equals

$$
-G:(H-G)=-\frac{1}{2}\left(|H|^{2}+|G|^{2}-|H-G|^{2}\right)+|G|^{2}=\frac{1}{2}|H-G|^{2}
$$

for $G, H \in \operatorname{SO}(n)$. Because $\left|F\left(x_{i}-\bar{x}\right)\right|^{2}=|F|^{2} / 4$ for every $i=1, \ldots, 2^{n}$ if $F$ is orthogonal, we see that $F \mapsto F^{\prime}$ maps $\mathrm{SO}(n)$ into $\mathrm{SO}(n)^{\prime}$ multiplying all distances by the factor $2^{(n-2) / 2}$. Thus $\left(H 5^{\prime}\right)$ gives the following more precise version of (H5):

$$
N^{\prime}\left(H^{\prime}\right)-N^{\prime}\left(G^{\prime}\right)-\nabla N^{\prime}\left(G^{\prime}\right):\left(H^{\prime}-G^{\prime}\right)=2^{1-n}\left|H^{\prime}-G^{\prime}\right|^{2}
$$

for $H^{\prime}, G^{\prime} \in \mathrm{SO}(n)^{\prime}$. Condition (H6) follows from the fact that in view of Theorem 3.2 , $N^{\prime}$ is a polynomial of degree $n$.

The following statement gives a sufficient condition for the validity of the Cauchy-Born rule.

Theorem 4.2. Suppose that the cell energy $E_{\text {cell }}$ satisfies $(\mathrm{H} 1)-(\mathrm{H} 4)$ and consider a discrete null Lagrangian $N^{\prime} \in C^{2}\left(\mathbb{R}^{n \times 2^{n}}\right)$ with the properties (H5)-(H6). Then there exists a convex function $H_{\text {cell }}: \mathbb{R}^{n \times 2^{n}} \rightarrow \mathbb{R}$, an $\varepsilon>0$, and an open neighbourhood $\mathcal{U}^{\prime}$ of $\mathrm{SO}(n)^{\prime}$ with the following properties:

(a) The function $H_{\text {cell }}$ is invariant under translations and strictly convex on the subspace of all discrete gradients (i.e., the orthogonal complement of the shift directions).

(b) We have

$$
E_{\text {cell }}=H_{\text {cell }}-\varepsilon N^{\prime} \quad \text { in } \mathcal{U}^{\prime}, \quad E_{\text {cell }} \geq H_{\text {cell }}-\varepsilon N^{\prime} \quad \text { on } \mathbb{R}^{n \times 2^{n}} .
$$


In particular, for each $\Lambda \subset \mathcal{L}$ and each $F \in \mathbb{M}^{n \times n}$ with $F^{\prime} \in \mathcal{U}^{\prime}$ the variational problem Minimize $\sum_{x \in \Lambda} E_{\text {cell }}\left(D^{\prime} y(x)\right) \quad$ subject to $\quad y(x)=F x$ for all $x \in \mathcal{L} \backslash \Lambda^{\circ}$

has the unique solution $y(x)=F x$ for all $x \in \mathcal{L}$.

Proof. We divide the proof into several steps.

Step 1. We first show that the assumptions (H5) and (H6) on $N^{\prime}$ imply that there exist constants $c_{N}>0$ and $C_{N}$ such that

$$
\begin{aligned}
N^{\prime}\left(H^{\prime}\right)-N^{\prime}\left(G^{\prime}\right)-D N^{\prime}\left(G^{\prime}\right) & \left(H^{\prime}-G^{\prime}\right) \geq c_{N}\left|H^{\prime}-G^{\prime}\right|^{2} \\
& -C_{N}\left(\operatorname{dist}^{2}\left(G^{\prime}, \operatorname{SO}(n)^{\prime}\right)+\operatorname{dist}^{n}\left(G^{\prime}, \operatorname{SO}(n)^{\prime}\right)\right) \\
& -C_{N}\left(\operatorname{dist}^{2}\left(H^{\prime}, \operatorname{SO}(n)^{\prime}\right)+\operatorname{dist}^{n}\left(H^{\prime}, \operatorname{SO}(n)^{\prime}\right)\right)
\end{aligned}
$$

for all $G^{\prime}, H^{\prime} \in V_{0}^{\perp}$, where $V_{0}^{\perp}$ is the orthogonal complement of the shift directions $\pi\left(\mathbb{R}^{n}\right)$ in $\mathbb{R}^{n \times 2^{n}}$. To see this, denote the left hand side in 4.1 by $f\left(G^{\prime}, H^{\prime}\right)$. In view of (H6), the expression $\left|f\left(G^{\prime}, H^{\prime}\right)\right|$ is of order $\left|G^{\prime}\right|^{n}+\left|H^{\prime}\right|^{n}$ near infinity and hence controlled by $\operatorname{dist}^{n}\left(G^{\prime}, \mathrm{SO}(n)^{\prime}\right)+\operatorname{dist}^{n}\left(H^{\prime}, \mathrm{SO}(n)^{\prime}\right)$ if the latter becomes large. We may therefore assume that $\left|G^{\prime}\right|^{2}+\left|H^{\prime}\right|^{2} \leq 2 R^{2}$ for a sufficiently large $R$. We notice that

$$
f\left(G^{\prime}, G^{\prime}\right)=0, \quad D f\left(G^{\prime}, G^{\prime}\right)=0 .
$$

Indeed, the first of these identities is immediate and it implies that $D f\left(G^{\prime}, G^{\prime}\right)\left(X^{\prime}, X^{\prime}\right)$ $=0$. In order to prove the second identity, we observe that $D f\left(G^{\prime}, G^{\prime}\right)\left(0^{\prime}, Y^{\prime}\right)=0$ and this establishes the assertion. Now choose $\bar{G}^{\prime}, \bar{H}^{\prime} \in \mathrm{SO}(n)^{\prime}$ such that

$$
\operatorname{dist}\left(G^{\prime}, \operatorname{SO}(n)^{\prime}\right)=\left|G^{\prime}-\bar{G}^{\prime}\right|, \quad \operatorname{dist}\left(H^{\prime}, \operatorname{SO}(n)^{\prime}\right)=\left|H^{\prime}-\bar{H}^{\prime}\right| .
$$

Since $\left|G^{\prime}\right|^{2}+\left|H^{\prime}\right|^{2} \leq 2 R^{2}$ we deduce by a Taylor expansion that

$$
\begin{aligned}
f\left(G^{\prime}, H^{\prime}\right) \geq & f\left(\bar{G}^{\prime}, \bar{H}^{\prime}\right)+D f\left(\bar{G}^{\prime}, \bar{H}^{\prime}\right)\left(G^{\prime}-\bar{G}^{\prime}, H^{\prime}-\bar{H}^{\prime}\right) \\
& -C\left(\left|G^{\prime}-\bar{G}^{\prime}\right|^{2}+\left|H^{\prime}-\bar{H}^{\prime}\right|^{2}\right)
\end{aligned}
$$

where the constant $C$ depends only on $R$. In view of 4.2 and the mean value theorem,

$$
|D f|\left(\bar{G}^{\prime}, \bar{H}^{\prime}\right)\left(G^{\prime}-\bar{G}^{\prime}, H^{\prime}-\bar{H}^{\prime}\right) \leq C\left|\bar{G}^{\prime}-\bar{H}^{\prime}\right|\left(\left|G^{\prime}-\bar{G}^{\prime}\right|^{2}+\left|H^{\prime}-\bar{H}^{\prime}\right|^{2}\right)^{1 / 2} .
$$

The assertion 4.1 follows now from (H5), Young's inequality $a b \leq \frac{\alpha}{2} a^{2}+\frac{1}{2 \alpha} b^{2}$ for $\alpha>0$, and the estimate $\left|G^{\prime}-H^{\prime}\right|^{2} \leq 3\left(\left|\bar{G}^{\prime}-\bar{H}^{\prime}\right|^{2}+\left|G^{\prime}-\bar{G}^{\prime}\right|^{2}+\left|H^{\prime}-\bar{H}^{\prime}\right|^{2}\right)$.

Step 2. For $\varepsilon>0$ we define

$$
g\left(F^{\prime}\right)=E_{\text {cell }}\left(F^{\prime}\right)+\varepsilon N^{\prime}\left(F^{\prime}\right) .
$$


The parameter $\varepsilon$ will be chosen below (see (4.4) and (4.6)). We now assert that there exists a neighbourhood $\mathcal{U}^{\prime}$ of $\operatorname{SO}(n)^{\prime}$ (which may depend on $\varepsilon$ ) and $c(\varepsilon)>0$ such that

$$
g\left(H^{\prime}\right)-g\left(G^{\prime}\right)-D g\left(G^{\prime}\right):\left(H^{\prime}-G^{\prime}\right) \geq c(\varepsilon)\left|H^{\prime}-G^{\prime}\right|^{2}
$$

for all $H^{\prime} \in V_{0}^{\perp}$ and $G^{\prime} \in V_{0}^{\perp} \cap \mathcal{U}^{\prime}$. This follows indeed by a standard perturbation and compactness argument. We first show the assertion for $G^{\prime} \in \mathrm{SO}(n)^{\prime}$. As in [FT02] we distinguish two cases.

Case 1: $\left|G^{\prime}-H^{\prime}\right|$ is small. In this case it suffices to verify that $D^{2} g\left(G^{\prime}\right)$ is positive definite (on $V_{0}^{\perp}$ ). Fix $G \in \mathrm{SO}(n)$. We denote by $P$ the orthogonal projection onto the tangent space $T_{G^{\prime}} \mathrm{SO}(n)^{\prime}$ and by $P^{\perp}$ the orthogonal projection onto the orthogonal complement of $T_{G^{\prime}} \mathrm{SO}(n)^{\prime} \oplus V_{0}$. Since $E_{\text {cell }}$ attains its minimum on $\mathrm{SO}(n)^{\prime}+V_{0}$, we have $D E_{\text {cell }}=0$ on $\mathrm{SO}(n)^{\prime}+V_{0}$, and thus by (H3) and frame indifference,

$$
D^{2} E_{\text {cell }}\left(G^{\prime}\right)\left(X^{\prime}, X^{\prime}\right)=D^{2} E_{\text {cell }}\left(G^{\prime}\right)\left(P^{\perp} X^{\prime}, P^{\perp} X^{\prime}\right) \geq c_{E}\left|P^{\perp} X^{\prime}\right|^{2} .
$$

On the other hand, 4.1 implies that for $X^{\prime} \in V_{0}^{\perp}$,

$$
D^{2} N^{\prime}\left(G^{\prime}\right)\left(X^{\prime}, X^{\prime}\right) \geq c_{N}\left|X^{\prime}\right|^{2}-C_{N}\left|P^{\perp} X^{\prime}\right|^{2} .
$$

If we choose

$$
\varepsilon \leq c_{E} / C_{N}
$$

then we conclude that

$$
D^{2} g\left(G^{\prime}\right)\left(X^{\prime}, X^{\prime}\right) \geq \varepsilon c_{N}\left|X^{\prime}\right|^{2}
$$

for all $X^{\prime} \in V_{0}^{\perp}$ and all $G^{\prime} \in \mathrm{SO}(n)^{\prime}$.

Case 2: $\left|G^{\prime}-H^{\prime}\right|$ is not small. For $G^{\prime} \in \mathrm{SO}(n)^{\prime}$ the left hand side in 4.3 reduces to

$$
E_{\text {cell }}\left(H^{\prime}\right)+\varepsilon\left(N^{\prime}\left(H^{\prime}\right)-N^{\prime}\left(G^{\prime}\right)-D N^{\prime}\left(G^{\prime}\right):\left(H^{\prime}-G^{\prime}\right)\right)
$$

since the elements in $\mathrm{SO}(n)^{\prime}$ are the minima of $E_{\text {cell }}$. We assert that for $H^{\prime} \in V_{0}^{\perp}$,

$$
E_{\text {cell }}\left(H^{\prime}\right) \geq \widetilde{c}_{E}\left(\operatorname{dist}^{2}\left(H^{\prime}, \operatorname{SO}(n)^{\prime}\right)+\operatorname{dist}^{n}\left(H^{\prime}, \mathrm{SO}(n)^{\prime}\right)\right)
$$

for some constant $\widetilde{c}_{E}>0$. Since by (H2) we have $E_{\text {cell }}\left(H^{\prime}\right)>0$ for $H^{\prime} \notin \operatorname{SO}(n)^{\prime}$, we only need to verify this inequality in the limiting cases $\operatorname{dist}\left(H^{\prime}, \operatorname{SO}(n)^{\prime}\right) \rightarrow \infty$ and $\operatorname{dist}\left(H^{\prime}, \mathrm{SO}(n)^{\prime}\right) \rightarrow 0$. In the former case the assertion follows from the growth condition $(\mathrm{H} 4)$, in the latter case it is implied by the coercivity condition (H3). Now 4.3 follows (for $G^{\prime} \in \mathrm{SO}(n)^{\prime}$ ) from 4.5) and 4.1) as long as

$$
\varepsilon \leq \widetilde{c}_{E} / C_{N}
$$

To obtain (4.3) in full generality we first choose $\varepsilon$ such that (4.4) and (4.6) hold. Then we conclude from Case 1 that $D^{2} g$ is positive definite (on $V_{0}^{\perp}$ ) in a neighbourhood $\mathcal{U}_{1}^{\prime}$ of 
$\mathrm{SO}(n)^{\prime}$. Hence there exists $\eta>0$ such that 4.3 holds for all $G^{\prime}$ in $\mathcal{U}_{1}^{\prime}$ and all $H^{\prime}$ with $\left|H^{\prime}-G^{\prime}\right|<\eta$. Now suppose $\left|H^{\prime}-G^{\prime}\right| \geq \eta$. If $G^{\prime} \in \operatorname{SO}(n)^{\prime}$ the inequality 4.3 holds by the considerations in Case 2 . Hence it continues to hold (with a slightly smaller constant on the right hand side) for $G^{\prime}$ in some neighbourhood $\mathcal{U}_{2}^{\prime}$ of $\operatorname{SO}(n)^{\prime}$. The assertion follows by taking $\mathcal{U}^{\prime}$ to be the intersection of the two neighbourhoods.

Step 3. We are now in a position to prove the assertion of the theorem. We define

$$
h\left(F^{\prime}\right)=\sup _{G^{\prime} \in \mathcal{U}^{\prime} \cap V_{0}^{\perp}}\left\{g\left(G^{\prime}\right)+D g\left(G^{\prime}\right):\left(F^{\prime}-G^{\prime}\right)+\frac{1}{2} c(\varepsilon)\left|F^{\prime}-G^{\prime}\right|^{2}\right\}
$$

for all $F^{\prime} \in V_{0}^{\perp}$. Then $h=g$ on $\mathcal{U}^{\prime} \cap V_{0}^{\perp}$ and $h \leq g$. Moreover, $h$ is uniformly convex on $V_{0}^{\perp}$ as a supremum over quadratic functions with fixed positive definite quadratic part. Extending $h$ so that it is constant in the shift directions $V_{0}$ we obtain $H_{\text {cell }}$.

Uniqueness of the minimizer $y$ is easy to see by the following argument. We know from the definition of the discrete null Lagrangian that

$$
\begin{aligned}
0 & \geq \sum_{x \in \Lambda} H_{\text {cell }}\left(D^{\prime} y(x)\right)-\sum_{x \in \Lambda} H_{\text {cell }}\left(F^{\prime}\right) \\
& \geq \sum_{x \in \Lambda} D H_{\text {cell }}\left(F^{\prime}\right)\left(D^{\prime} y(x)-F^{\prime}\right)=D H_{\text {cell }}\left(F^{\prime}\right)\left(\sum_{x \in \Lambda} D^{\prime} y(x)-F^{\prime}\right)=0 .
\end{aligned}
$$

Since we consider discrete gradients only, the second inequality is sharp whenever $D^{\prime} y(x)$ $\neq F^{\prime}$. Thus we conclude that $z(x):=y(x)-F x$ satisfies $D^{\prime} z(x)=0$ for every $x \in \Lambda$. For $x \notin \Lambda$ we have $x+x_{i} \notin \Lambda^{\circ}$ and thus by the boundary conditions

$$
z(x)=z\left(x+x_{i}\right)=0, \quad \forall x \in \mathcal{L} \backslash \Lambda, i=1, \ldots, 2^{n} .
$$

Hence $D^{\prime} z(x)=0$ for all $x \in \mathcal{L}$. This implies that there exist $c(x)$ such that $z\left(x+x_{i}\right)=$ $c(x)$ for all $i$. Hence $z$ is constant and by 4.7) we conclude that $z=0$ on $\mathcal{L}$.

\section{Mass-spring models}

We now briefly indicate how mass-spring models fit into the above framework. As a warm-up consider the situation discussed in [FT02], i.e., $n=m=2$ and an energy involving nearest and next-nearest neighbour interactions

$$
E(y)=\sum_{\left|x-x^{\prime}\right|=1} V_{1}\left(y(x)-y\left(x^{\prime}\right)\right)+\sum_{\left|x-x^{\prime}\right|=\sqrt{2}} V_{2}\left(y(x)-y\left(x^{\prime}\right)\right) .
$$

Since each nearest neighbour bond belongs to two unit cells the corresponding cell energy (which involves the values $y\left(x_{1}\right), \ldots, y\left(x_{4}\right)$ of $y$ at the corners of the unit square) is given by

$$
E_{\text {cell }}(y)=\frac{1}{2} \sum_{\left|x_{i}-x_{j}\right|=1} V_{1}\left(y\left(x_{i}\right)-y\left(x_{j}\right)\right)+\sum_{\left|x_{i}-x_{j}\right|=\sqrt{2}} V_{2}\left(y\left(x_{i}\right)-y\left(x_{j}\right)\right)
$$


For general finite-range interactions it is in general not possible to write the total energy as a sum of contributions each of which only involves values on a single cell. This difficulty can, however, easily be overcome by passing to larger building blocks. Thus instead of $E_{\text {cell }}: \mathbb{R}^{n \times 2^{n}} \rightarrow[0, \infty)$ we consider

$$
E_{A}: \mathbb{R}^{n \times A} \rightarrow[0, \infty)
$$

where $A \subset \mathcal{L}$ is a finite set which satisfies

$$
x_{i} \in A \quad \text { for } i=1, \ldots, 2^{n},
$$

where the $x_{i}$ are the vertices of the (cubic) unit cell. We adjust the definition of interior points accordingly by defining

$$
\Lambda^{\circ}=\Lambda_{A}^{\circ}:=\{z \in \Lambda: z-a \in \Lambda \forall a \in A\} .
$$

Note that the boundary $\Lambda \backslash \Lambda^{\circ}$ can now consist of several layers of lattice points-this is, however, in full accordance with the physical intuition of a finite-range interaction. As before we define the constant embedding $\pi$ and the subspace of shift directions $V_{0}$ (whose orthogonal complement is the space of discrete gradients) by considering vectors with coordinates in $\mathbb{R}^{n}$ but with index set $A$ instead of $\left\{1, \ldots, 2^{n}\right\}$.

For a general map $y: \mathcal{L} \rightarrow \mathbb{R}^{n}$ we define the discrete gradient at the point $x \in \mathcal{L}$ by

$$
\left(D_{A}^{\prime} y\right)_{a}(x)=y(x+a)-\frac{1}{\operatorname{card}(A)} \sum_{b \in A} y(x+b), \quad a \in A .
$$

In particular,

$$
\bar{a}=\frac{1}{\operatorname{card}(A)} \sum_{a \in A} a, \quad \text { and } \quad F_{a}^{\prime}=F(a-\bar{a}) \quad \text { for } a \in A, F \in \mathbb{M}^{n \times n}
$$

is the discrete gradient of the affine map $x \mapsto F x$, constant on all of $\mathcal{L}$.

The assumptions $(\mathrm{H} 1), \ldots,(\mathrm{H} 4)$ then remain completely unchanged, just with $E_{A}$ substituted for $E_{\text {cell }}$. It has to be observed, however, that the verification of (H3) becomes more difficult with growing size of $A$.

For a map $y: A \rightarrow \mathbb{R}^{n}$ we consider again its coordinatewise affine extension to $[0,1]^{n}$ and use this extension as before to transform null Lagrangians for the continuous system into discrete null Lagrangians on $\left(\mathbb{R}^{n}\right)^{A}$. These discrete null Lagrangians only involve the values of $y$ at the vertices of the unit cell (and not at all points of $A$ ) but this turns out to be enough (essentially, we only need the discrete null Lagrangian to control rigid motions and these are already determined by their values on the vertices of the unit cell). The crucial step in the argument is, as before, that for any $x \in \Lambda^{\circ}$ we have $x-x^{i} \in \Lambda$, $i=1, \ldots, 2^{n}$, and hence

$$
x \in \operatorname{int}\left(\bigcup_{i}\left(x-x^{i}\right)+[0,1]^{n}\right) \subset \operatorname{int}\left(\Lambda+[0,1]^{n}\right) .
$$

We also note that (H5) and (H6) are again satisfied for the discrete null Lagrangian $N^{\prime}$ obtained from the determinant. 
Theorem 5.1. Suppose that the cell energy $E_{A}$ satisfies $(\mathrm{H} 1)-(\mathrm{H} 4)$ and consider a discrete null Lagrangian $N^{\prime} \in C^{2}\left(\mathbb{R}^{n \times A}\right)$ with the properties (H5)-(H6). Then there exists a convex function $H_{A}: \mathbb{R}^{n \times A} \rightarrow \mathbb{R}$, an $\varepsilon>0$, and an open neighbourhood $\mathcal{U}^{\prime}$ of $\mathrm{SO}(n)^{\prime}$ with the following properties:

(a) The function $H_{A}$ is invariant under translations (i.e., under adding shift directions) and strictly convex on the subspace of all discrete gradients.

(b) We have

$$
E_{A}=H_{A}-\varepsilon N^{\prime} \quad \text { in } \mathcal{U}^{\prime}, \quad E_{A} \geq H_{A}-\varepsilon N^{\prime} \quad \text { on } \mathbb{R}^{n \times A} .
$$

In particular, for each $\Lambda \subset \mathcal{L}$ and $F \in \mathbb{M}^{n \times n}$ with $F^{\prime} \in \mathcal{U}^{\prime}$ the variational problem

$$
\text { Minimize } \sum_{x \in \Lambda} E_{A}\left(D_{A}^{\prime} y\right)(x) \quad \text { subject to } y(x)=F x \text { for all } x \in \mathcal{L} \backslash \Lambda^{\circ}
$$

has the unique solution $y(x)=F x$ for all $x \in \mathcal{L}$.

The proof of the theorem is identical with the one of Theorem 4.2 .

\section{Appendix}

For the convenience of the reader we give a proof for the characterization of null Lagrangians which does not go through the Euler-Lagrange equations (and which is shorter than the one in [Da89]). For proofs starting from the Euler-Lagrange equations see [La42, Er62, Ed62, DF64, Ru66, Ru74], where also more general Lagrangians of the form $N(x, u, \nabla u)$ are treated.

Theorem 6.1. Let $N: \mathbb{M}^{m \times n} \rightarrow \mathbb{R}$ be a continuous null Lagrangian. Then $N(F)$ is the sum of a constant term and a linear combination of minors (subdeterminants) of $F$.

Proof. First, considering essentially one-dimensional test functions $\phi$ of the form $\phi(x)=$ $\varepsilon \eta(x) a h(x \cdot b / \varepsilon)$, where $a \in \mathbb{R}^{m}, b \in \mathbb{R}^{n}$ and where $\eta$ is a smooth cut-off function, we infer that $N$ is affine along any rank-one line in $\mathbb{M}^{m \times n}$, i.e., on all lines of the form $t \mapsto F+t a \otimes b$ (see, e.g., [Da89]; in fact, if one uses test functions which take only finitely many gradients, then continuity of $N$ is not needed to reach the conclusion [Fo88, Mü99|).

It follows that the second (distributional) derivative of $N$ in any rank-one direction $A$ is zero. To see this we write $A^{\perp}$ for the subspace of $\mathbb{M}^{m \times n}$ perpendicular to $A$ and we obtain, for $\varphi \in C_{0}^{\infty}\left(\mathbb{M}^{m \times n}\right)$ and $\psi_{F}(t):=\varphi(F+t A)$,

$$
\begin{aligned}
\left\langle D^{2} N(\cdot)(A, A), \varphi\right\rangle & =\int_{\mathbb{M}^{m} m \times n} N(G) D^{2} \varphi(G)(A, A) d G \\
& =\int_{A^{\perp}} \int_{\mathbb{R}} N(F+t A) D^{2} \varphi(F+t A)(A, A)|A| d t d \mathcal{H}^{n-1}(F), \\
& =\int_{A^{\perp}} \int_{\mathbb{R}} N(F+t A) \frac{d^{2}}{d t^{2}} \psi_{F}(t)|A| d t d \mathcal{H}^{n-1}(F)=0,
\end{aligned}
$$

since the inner integral vanishes as $t \mapsto N(F+t A)$ is affine. 
Taking further derivatives of the distribution $D^{2} N(\cdot)(A, A)$ we get

$$
D^{k} N(\cdot)\left(A, A, H_{3}, \ldots, H_{k}\right)=0 \quad \text { if } \operatorname{rank}(A) \leq 1 .
$$

The crucial observation is that this implies that

$$
D^{k} N(\cdot)(F, \ldots, F)=0 \quad \text { if } \operatorname{rank}(F)<k .
$$

Indeed, $F$ can be written as a sum of at most $k-1$ rank-one matrices, $F=\sum_{i=1}^{k-1} A_{i}$, and by multilinearity the left hand side of (6.2) can be expressed as a sum of terms of the form $D^{k} N(\cdot)\left(A_{j_{1}}, \ldots, A_{j_{k}}\right)$. Thus at least two of the indices $j_{1}, \ldots, j_{k}$ must coincide and by 6.1 and the symmetry of the derivative all these terms must vanish. Applying this for $k=d+1$, where $d=\min (m, n)$, we see that $D^{d+1} N(\cdot)(F, \ldots, F)=0$ for all $F \in \mathbb{M}^{m \times n}$. Since $D^{d+1} N(\cdot)$ is a symmetric multilinear map this implies that it vanishes identically (see Proposition 6.2 below). Hence $N$ is a polynomial of degree at most $d$ and the previous identities hold also pointwise. From Taylor's formula we obtain

$$
N(F)=\sum_{k=0}^{d} P_{k}(F) \quad \text { with } \quad P_{k}(F)=\frac{1}{k !} D^{k} N(0)(F, \ldots, F),
$$

the $P_{k}$ 's being homogeneous polynomials of degree $k$ over $\mathbb{M}^{m \times n}$.

We want to show that $P_{k}$ contains only those products of matrix entries that also occur in the definition of the subdeterminants of size $k$. To do so we define for arbitrary subsets $I \subset\{1, \ldots, m\}$ and $J \subset\{1, \ldots, n\}$ the matrix $F_{I, J}$ by

$$
\left(F_{I, J}\right)_{i, j}= \begin{cases}F_{i, j} & \text { if } i \in I \text { and } j \in J \\ 0 & \text { else. }\end{cases}
$$

If $I=\left\{\alpha_{1}, \ldots, \alpha_{k}\right\}$ with $1 \leq \alpha_{1}<\cdots<\alpha_{k} \leq m$ and $J=\left\{\beta_{1}, \ldots, \beta_{k}\right\}$ with $1 \leq \beta_{1}<$ $\cdots<\beta_{k} \leq n$ then we $\operatorname{define} \operatorname{det}_{k} F_{I, J}$ as the determinant of the $k \times k$ matrix $G$ given by $G_{l, m}=F_{\alpha_{l}, \beta_{m}}=\left(F_{I, J}\right)_{\alpha_{l}, \beta_{m}}$.

The polynomial $P_{k}(F)$ is a linear combination of products $F_{\alpha_{1}, \beta_{1}} F_{\alpha_{2}, \beta_{2}} \cdots F_{\alpha_{k}, \beta_{k}}$. We regroup the sum by collecting those terms for which the indices $\alpha_{i}$ and $\beta_{i}$ lie in fixed subsets $I$ and $J$ of $\{1, \ldots, m\}$ and $\{1, \ldots n\}$, respectively. This yields

$$
P_{k}(F)=\sum_{\substack{I \subset\{1, \ldots, m\} \\ J \subset\{1, \ldots, n\}}} \sum_{\substack{\alpha=\left(\alpha_{1}, \ldots, \alpha_{k}\right) \\\left\{\alpha_{1}, \ldots, \alpha_{k}\right\}=I}} \sum_{\substack{\beta=\left(\beta_{1}, \ldots, \beta_{k}\right) \\\left\{\beta_{1}, \ldots, \beta_{k}\right\}=J}} \tilde{c}_{\alpha, \beta} F_{\alpha_{1}, \beta_{1}} F_{\alpha_{2}, \beta_{2}} \cdots F_{\alpha_{k}, \beta_{k}} .
$$

We next show that if $\operatorname{card}(I)<k$ or $\operatorname{card}(J)<k$ then the inner double sum is zero. We have $\operatorname{rank}\left(F_{I, J}\right)<k$ and in view of 6.2 and 6.3 this yields

$$
\sum_{\substack{\alpha=\left(\alpha_{1}, \ldots, \alpha_{k}\right) \\\left\{\alpha_{1}, \ldots, \alpha_{k}\right\} \subset I}} \sum_{\substack{\beta=\left(\beta_{1}, \ldots, \beta_{k}\right) \\\left\{\beta_{1}, \ldots, \beta_{k}\right\} \subset J}} \tilde{c}_{\alpha, \beta} F_{\alpha_{1}, \beta_{1}} F_{\alpha_{2}, \beta_{2}} \cdots F_{\alpha_{k}, \beta_{k}}=P_{k}\left(F_{I, J}\right)=0
$$


for all $F \in \mathbb{M}^{m \times n}$. From this we conclude, by separate induction on card $(I)$ and $\operatorname{card}(J)$, that also

$$
\sum_{\substack{\alpha=\left(\alpha_{1}, \ldots, \alpha_{k}\right) \\\left\{\alpha_{1}, \ldots, \alpha_{k}\right\}=I}} \sum_{\substack{\beta=\left(\beta_{1}, \ldots, \beta_{k}\right) \\\left\{\beta_{1}, \ldots, \beta_{k}\right\}=J}} \tilde{c}_{\alpha, \beta} F_{\alpha_{1}, \beta_{1}} F_{\alpha_{2}, \beta_{2}} \cdots F_{\alpha_{k}, \beta_{k}}=0
$$

if $\operatorname{card}(I)<k$ or $\operatorname{card}(J)<k$.

Thus we only need to consider index sets $I$ and $J$ which contain exactly $k$ elements. Regrouping terms which are obtained by permutation within a fixed index set we see that

$$
P_{k}(F)=\sum_{\substack{1 \leq \alpha_{1}<\cdots<\alpha_{k} \leq m \\ 1 \leq \beta_{1}<\cdots<\beta_{k} \leq n}} P_{\alpha, \beta}(F),
$$

with

$$
P_{\alpha, \beta}(F)=\sum_{\sigma \in S_{k}} c_{\alpha, \beta}^{\sigma} F_{\alpha_{1}, \beta_{\sigma_{1}}} F_{\alpha_{2}, \beta_{\sigma_{2}}} \cdots F_{\alpha_{k}, \beta_{\sigma_{k}}},
$$

where the sum is taken over the group $S_{k}$ of all permutations of $\{1, \ldots, k\}$. Here $c_{\alpha, \beta}^{\sigma}=$ $\sum_{\tau \in S_{k}} \tilde{c}_{\tau(\alpha),(\tau \circ \sigma)(\beta)}$, and $\tau(\alpha)_{i}=\alpha_{\tau_{i}}$.

To conclude we only need to show that $P_{\alpha, \beta}(F)$ is a multiple of $\operatorname{det}_{k} F_{I, J}$. To this end we consider the multilinear form

$$
Q_{\alpha, \beta}\left(v^{1}, \ldots, v^{k}\right)=\sum_{\sigma \in S_{k}} c_{\alpha, \beta}^{\sigma} v_{1}^{\sigma_{1}} v_{2}^{\sigma_{2}} \cdots v_{k}^{\sigma_{k}}
$$

on $\left(\mathbb{R}^{k}\right)^{k}$. Let $F_{v}$ denote the matrix with columns $v^{1}, \ldots, v^{k}$, i.e., $F_{v}=\sum_{i, j=1}^{k} v_{i}^{j} e_{\alpha_{i}}$ $\otimes e_{\beta_{j}}$. Then

$$
Q_{\alpha, \beta}\left(v^{1}, \ldots, v^{k}\right)=P_{\alpha, \beta}\left(F_{v}\right)=P_{k}\left(F_{v}\right)
$$

and we see from 6.2 and 6.3 that $Q_{\alpha, \beta}\left(v^{1}, \ldots, v^{k}\right)=0$ if $\operatorname{dim}\left(\operatorname{span}\left(\left\{v^{1}, \ldots, v^{k}\right\}\right)\right)=$ $\operatorname{rank}\left(F_{v}\right)<k$. By multilinearity this implies that $Q_{\alpha, \beta}$ is antisymmetric (indeed we have $\left.0=Q\left(v^{1}+v^{2}, v^{1}+v^{2}, \ldots\right)=Q\left(v^{1}, v^{2}, \ldots\right)+Q\left(v^{2}, v^{1}, \ldots\right)\right)$. Now every antisymmetric multilinear form is a multiple of the determinant, i.e.,

$$
Q_{\alpha, \beta}\left(v^{1}, \ldots, v^{k}\right)=\hat{c}_{\alpha, \beta} \operatorname{det}\left(v^{1}, \ldots, v^{k}\right) \quad \text { for all } v \in\left(\mathbb{R}^{k}\right)^{k} .
$$

Thus $P_{\alpha, \beta}(F)=\hat{c}_{\alpha, \beta} \operatorname{det}_{k}\left(F_{I, J}\right)$ if we choose $I=\left\{\alpha_{1}, \ldots, \alpha_{k}\right\}$ and $J=\left\{\beta_{1}, \ldots, \beta_{k}\right\}$, and the proof is finished.

We have used the following well-known fact.

Proposition 6.2. Let $V$ be a finite-dimensional vector space and let $M$ be a symmetric multilinear map on $V^{k}$ such that $M(F, \ldots, F)=0$ for all $F \in V$. Then $M$ vanishes identically.

This is obvious for $k=1$. To carry out the induction step from $k$ to $k+1$ one fixes $G \in V$, defines the $k$-linear form $M^{\prime}\left(F_{1}, \ldots, F_{k}\right):=M\left(F_{1}, \ldots, F_{k}, G\right)$ and uses the identity

$$
M^{\prime}(F, \ldots, F)=\left.\frac{1}{k+1} \frac{d}{d s}\right|_{\mid s=0} M(F+s G, \ldots, F+s G)=0 .
$$


Acknowledgements. G.D. gratefully acknowledges partial support by the Max Planck Society and by the NSF through grants DMS0104118 and DMS0405853; S.C. and S.M. were supported by the DFG priority programme SPP 1095; S.C., S.M and B.K. were also supported by the EU programme MRTN-CT-2004-505226.

\section{References}

[Ba77] Ball, J. M.: Convexity conditions and existence theorems in nonlinear elasticity. Arch. Rat. Mech. Anal. 63, 337-403 (1977) Zbl 0368.73040 MR 0475169

[BCO81] Ball, J. M., Currie, J. C., Olver, P. C.: Null Lagrangians, weak continuity and variational problems of arbitrary order. J. Funct. Anal. 41, 135-174 (1981) Zbl 0459.35020 MR 0615159

[BKK00] Ball, J. M., Kirchheim, B., Kristensen, J.: Regularity of quasiconvex envelopes. Calc. Var. Partial Differential Equations 11, 333-359 (2000) Zbl 0972.49024 MR 1808126

[BJ87] Ball, J. M., James, R. D.: Fine phase mixtures as minimizers of energy. Arch. Rat. Mech. Anal. 100, 13-52 (1987) Zbl 0629.49020 MR 0906132

[Bh03] Bhattacharya, K.: Microstructure of Martensite. Oxford Univ. Press (2003)

[BCZZ04] Bhattacharya, K., Conti, S., Zanzotto, G., Zimmer, J.: Crystal symmetry and the reversibility of phase transformations. Nature 428, 55-59 (2004)

[CK88] Chipot, M., Kinderlehrer, D.: Equilibrium configurations of crystals. Arch. Rat. Mech. Anal. 103, 237-277 (1988) Zbl 0673.73012 MR 0955934

[CZ04] Conti, S., Zanzotto, G.: A variational model for reconstructive phase transformations in crystals, and their relation to dislocations and plasticity. Arch. Rat. Mech. Anal. 173, 69-88 (2004) Zbl pre02113861 MR 2073505

[Da89] Dacorogna, B.: Direct Methods in the Calculus of Variations. Springer (1989) Zbl 0703.49001 MR 0990890

[DF64] De Franchis, M.: La più generale funzione d'invarianza per criteri sufficienti di minimo con condizioni di Dirichlet per integrali pluridimensionali del primo ordine dipendenti da un vettore a più componenti. Atti Accad. Naz. Lincei Rend. Cl. Sci. Fis. Mat. Natur. 37, no. 8, 129-140 (1964) Zbl 0128.10004 MR 0176366

[Do03] Dolzmann, G.: Variational Methods for Crystalline Microstructure-Analysis and Computation. Springer (2003) Zbl 1016.74002 MR 1954274

[EM05] E, W., Ming, P. B.: in preparation.

[Ed62] Edelen, D. G. B.: The null set of the Euler-Lagrange operator. Arch. Rat. Mech. Anal. 11, 117-121 (1962) Zbl 0125.33002 MR 0150623

[Er62] Ericksen, J. L.: Nilpotent energies in liquid crystal theory. Arch. Rat. Mech. Anal. 10, 189-196 (1962) Zbl 0109.23002 MR 0169513

[Er82] Ericksen, J.: The Cauchy and Born hypotheses for crystals. In: Phase Transitions and Material Instabilities in Solids, M. Gurtin (ed.), Academic Press, 61-77 (1984) Zbl 0567.73112 MR 0802219

[Er97] Ericksen, J.: Equilibrium theory for X-ray observations of crystals. Arch. Rat. Mech. Anal. 139, 181-200 (1997) Zbl 0910.73056 MR 1478777

[Fo87] Fonseca, I.: Variational methods for elastic crystals. Arch. Rat. Mech. Anal. 97, 189220 (1987) Zbl 0611.73023 MR 0862547

[Fo88] Fonseca, I.: The lower quasiconvex envelope of the stored energy function for an elastic crystal. J. Math. Pures Appl. 67, 175-195 (1988) Zbl $0718.73075 \mid$ MR 0949107 
[FT02] Friesecke, G., Theil, F.: Validity and failure of the Cauchy-Born rule in a two-dimensional mass-spring lattice. J. Nonlinear Sci. 12, 445-478 (2002) Zbl pre01903651 MR 1923388

[JH00] James, R. D., Hane, K. F.: Martensitic transformations and shape-memory materials. Acta Materialia 48, 197-222 (2000)

[La42] Landers, A. W.: Invariant multiple integrals in the calculus of variations. In: Contributions to the Calculus of Variations, 1938-1941, Univ. Chicago Press, 184-189 (1942) Zbl 0063.03441 MR 0006821

[Mo52] Morrey, C. B.: Quasi-convexity and the lower semicontinuity of multiple integrals. Pacific J. Math. 2, 25-53 (1952) Zbl 0046.10803 MR 0054865

[Mü99] Müller, S.: Variational methods for microstructure and phase transitions. In: Proc. CIME Summer School on Calculus of Variations and Geometric Evolution Problems, F. Bethuel et al. (eds.), Lecture Notes in Math. 1713, Springer, 85-210 (1999) Zbl 0968.74050 MR 1731640

[Pa80] Parry, G. P.: On phase transitions involving internal strain. Int. J. Solids Structures 17, 361-378 (1980) Zbl 0464.73004 MR 0611096

[PZ03] Pitteri, M., Zanzotto, G.: Continuum Models for Phase Transitions and Twinning in Crystals. Chapman \& Hall (2003) Zbl 1019.80001 MR 1996833

[Ru66] Rund, H.: The Hamilton-Jacobi Theory in the Calculus of Variations. Van Nostrand (1966) Zbl 0141.10602 MR 0230189

[Ru74] Rund, H.: Integral formulae associated with the Euler-Lagrange operator of multiple integrals, Aequationes Math. 11, 212-229 (1974) Zbl 0293.49001 MR 0361971

[Za92] Zanzotto, G.: On the material symmetry group of elastic crystals and the Born rule. Arch. Rat. Mech. Anal. 121, 1-36 (1992) Zbl 0781.73005 MR 1185569 